\title{
Burkholderia Cepacia An Emerging Cause Of Septicemia- An Outbreak In A Neonatl Intensive Care Unit From A Tertiary Care Hospital Of Central India.
}

\author{
Dr Swati M. Bhise, Dr Vaishali A. Rahangdale, Dr M.S. Qazi. \\ Department Of Microbiology \& Department Of Paediatric Government Medical College,Nagpur.
}

\begin{abstract}
We report an outbreak of blood stream infection from a neonatal intensive care unit in the month of April 2013. 10 neonates were clinically diagnosed as septicemia. In all the neonates blood culture was positive for B.cepacia. All the patients had indwelling intravenous catheters. in 4 patients B, cepacia was isolated from the culture of catheter tips. Though we did not identify the source of the organism in other 6 cases, our findings support the hypothesis that cross contamination may be the contributing factor. We recommend that Burkholderia cepacia should always be considered as an emerging cause of gram negative septicemia in neonates \& should be treated immediately to reduce the mortality due this multidrugresistant bug.
\end{abstract}

\section{Introduction}

Burkholderia cepacia complex is a phytopathogen first described in 1950 as causative agent of onion $\operatorname{rot}^{1}$, is increasingly recognized as an important cause of morbidity \& mortality associated with infections in immunocompromised patients and hospitalized patients probably because of intrinsic resistance to antimicrobial agents ${ }^{2,3}$. Burkholderia cepacia is an aerobic ,gram negative, nonfermentative bacillus widely distributed in the environment, including water,soil, fruits and vegetables ${ }^{4}$. They cause a wide variety of infections ranging from superficial to deep seated \& disseminated infections such as pneumonia, (especially in patients with cystic fibrosis),meningitis, peritonitis(in patients undergoing peritoneal dialysis), septicemia and bronchiectasis ${ }^{2}$. BCC though reported worldwide has not been reported from India and isolation of only two strains has been published from another tertiary care centre in India ${ }^{5}$. Here we report a outbreak of BCC from a neonatal intensive care unit within a period of a month.

\section{Material \& Methods}

Ten Premature newborns aged from 5 to 33 days were referred from the labour room 0f hospital to the neonatal intensive care unit as cases of clinically diagnosed, neonatal septicemia in the month of March- April 2013 . Blood culture of these patients were carried out in the Bacteriology laboratory of the department of Microbiology Government Medical College Nagpur as a part of routine diagnostic services . Blood culture was performed in BACT/ Alert 3D (Biomeriux). Blood culture positivity was detected within 48 hours in all the samples.These positive samples were subcultured by convetional methods on sheep Blood agar, chocolate agar and MacConkey's agar; incubated for 48 hours aerobically in $5-10 \% \mathrm{co}_{2}$ at $37^{\circ} \mathrm{c}$. After 24 hours typical large ,circular, low convex ,moist $\beta$ haemolytic colonies was observed on Blood agar \& non-lactose fermenting colonies on MacConkey's agar. In positive cases the isolates were tentatively identified to the species level by conventional biochemical tests ${ }^{22}$. Gram negative, motile, oxidase positive isolates were confirmed by Mini API identification system (Biomeriux).Antibiotic sensitivity testing was done by Kirby- Bauer disc diffusion method as per Clinical Laboratory Standard Institute, $2010{ }^{10}$.All the patients had biological signs of sepsis (reduced platelate count and elevated C-reactive protein,raised WBC count) and 5 patients had clinical signs of septic shock (fever $\geq 38 \mathrm{c}$ ).

Environmental and epidemiological investigations were conducted to identify the source and route of infection.Environmental samples included tap water, sink drains, incubator surfaces ,respiratory devices, suction machine, suction catheter,surface swabs and antiseptic solutions.None of the Environmental samples as well as antiseptic products grew B.cepacia.Samples of intravenous solution administered to all the patients were collected from the stocks of each ward \& from control pharmacy (one sample of each different lot). They were inoculated in blood culture bottles in Bact/ Alert 3D (Biomeriux) for 6 days. None of the IV solution samples grew B.cepacia.All the patients had central venous catheter for parenteral nutrition, 4 out of 10 patients catheter culture incriminated as the source of bacteremia. The catheter tips were cultured on conventional media and the catheter tips grew B.cepacia within 48 hours. The isolates were further identified by conventional methods as well as confirmed by Mini API system. Urine culture of all the patients were negative . 
All the infants recovered on antibiotic $t$.tracheal and feaceal sampling was done to determine colonization status.None of the patients had tracheal or Digestive colonization before or after the onset of bacteremia.The cases clustered in a very short period pointing to direct access of the pathogen to the blood stream

.Five patients who were in septic shock expired within 3 days of admission.So we could not reisolate the pathogen from those patients.In other patients we reisolated Burkholderia cepacia from blood samples.Other five patients responded well to the therapy chosen according to in vitro susceptibility .

\section{Discussion}

Septicemia is when bacteria are introduced into the the blood and multiply at a rate greater than the body can defend .Gram negative bacteria especially E.coli,Klebsiella are the major causes of septicemia in children ${ }^{24}$. However,Burkholderia cepacia has been rarely reported from blood stream infections. Unlike Pseudomonas aeruginosa which may be carried by around 10\% humans (e.g. as a gut colonizer),BCC bacteria have not yet been recovered from human sources other than the sites of infection. Therefore, in the absence of patient to patient transmission in Cystic fibrosis infection, the natural environment must be the reservoir of BCC infection ${ }^{21}$. Burkhoideria cepacia is a non-fermentative gram negative bacterium that causes severe pulmonary infections in Cystic fibrosis cases \& other immunocompromised hosts.however the bacterium has been isolated from Urinary tract, bloodstream, and nosocomial outbreaks ${ }^{23}$.Isolation \& identification of .Burkhoideria cepacia can be difficult due to the fact that it is slow growing and sometimes the biochemical reactions are variable.

$\mathrm{BCC}$ is being increasingly recognised as an important pathogen of humans in both immunocompromised and hospitalized patients who were infected by contact with contaminated equipment during hospitalization ${ }^{2}$.We investigated 10 cases of of neonatal septicemia in which B.cepacia was recovered from the blood culture of neonates admitted in NICU of Govt .Medical College \& Hospital,Nagpur.The ability of B.cepacia to colonize indwelling intravenous catheters and propagate there is likely contributed to this outbreak ${ }^{11,12}$.The presence of organic matter on indwelling catheters, especially those that have been implanted for long periods of time creates a favourable environment for bacterial colonization and the establishment of a microbial film ${ }^{13}$. This risk is clearly enhanced if these catheters are exposed to contaminated medications.

BCC often are resistant to disinfectants and antiseptic solutions, ${ }^{14,15,16}$ have unique ability to survive in standing water , and occassionaly are a cause of health care associated infection as a result of contamination ${ }^{17,18,19}$.

BCC bacteremia is most often cather related and polymicrobial has been reported in Cancer patients( and in patients undergoining haemodialysis ${ }^{25}$.

In one large hospital 74 patients at two hospital in Arizona had B.cepacia bacteremia during a 2 year period ${ }^{16}$.None of the patients had medical conditions associated with infection with B.cepacia .In these cases infection was associated with contaminated mouthwash.Due to high intrinsic resistance of the BCC to antibiotics and antimicrobial compounds, all of these infections can prove very difficult to treat and may be fatal ${ }^{3,20}$.In India ,there are no precise reports of the prevalence of BCC infection due to lack of specific laboratory tests and in most cases these bacteria have been ambiguously reported as non fermentative gram negative bacilli or simply as Pseudomonas spp.For this reason reports of diseases due to these organisms are rare and it has been reported from few tertiary care centres in North India ${ }^{6,7,8,9}$.A sudden upsurge of BCC has been observed in non-cystic -septicemic patients of PGIMER, Chandigarh and Escorts heart Institute and Research Centre (EHIRC)Delhi ${ }^{6}$.At PGIMER, has been isolated from the patients admitted in different wards with increased isolation from children in advanced paediatric centre ${ }^{6,8}$. BCC has been recognized as the third most common non-fermenter over the last six years in PGIMER after P.aeruginosa and A.calcoaceticusbaumanni complex ${ }^{6,7,8,9}$.Various outbreaks \& pseudooutbreaks of BCC septicemia have also been documented ${ }^{2}$.Outbreaks have been reported from nebulisers, chlorhexidine solution,alcohol free mouthwash,multidose albuterol vials,indigo-carmine dye in enteral feeding,tap water,bottled water,cosmetics,napkins,nasal sprays and ultrasound gels ${ }^{2,25,26}$.

A significant problem in managing $\mathrm{BCC}$ infected patients is the antimicrobial resistance and lack of newer effective antibiotics.BCC is intrinsically resistant to antimicrobial agents such as aminoglycosides,first and second generation cephalosporins, antipseudomonal penicillins and polymyxins. These various groups are commonly used in Pseudomonas infections, and the value of proper identification of BCC comes to the forefront 2,22. As per the CLSI 2010 guidelines, the drugs recommended against BCC are ceftazidime,minocycline, meropenem and cotrimoxazole ${ }^{2.22}$. There were several limitations to our analysis that we could not reisolate the organism as 8 patients were expired in NICU.We couldnot trace the source of infection in 6 patients.

\section{Conclusion}

Many health care providers prescribe antibiotics without culturing focal site of infection this lead to multidrugresistant bacteria.Our investigations also highlights the importance of critically evaluating the need to 
keep an implanted catheter in place once treatment is complete .Burkholderia cepacia bacteremia should be considered in febrile patients with nosocomial infections,especially those who have an indwelling catheter, are on ventilators, or have immune dysfunction. Burkholderia cepacia should always be kept in mind as a cause of Septicemia in neonates so as to reduce mortality due this multidrug resistant organism. Strict hospital surveillanace and aseptic precautions should be taken to avoid hospital acquired cross contamination.

\section{References-}

[1]. Burkholder WH.Sour skin,abacterial rot of onion bulbs.Phytopathology 1950:40, 115- 7.

[2]. Lipuma JJ,CurieBJ, Lum GD,Vandamme PAR.

[3]. Burkholderia,Stenotrophomonas,Ralstonia,Cupriavidus,Pandoraea,Brevudimonas,Comamonas,Delftia, and Acidovorax.In Murray PR, Baron EJ,Jorgensen JH,Pfaller MA, and Yolken RH,editors Manual of Clinical Microbiology,ASM Press, Washington ,DC 2007:749-69.

[4]. Hancock REW,Resistance mechanism in Pseudomonas aeruginosa and other non-fermenter gram negative bacteria.Clin Infect Dis

[5]. Editorial,Alicia Mangram,William R.Jarvis.Nosocomial outbreaks and pseudooutbreaks,Infection control and hospital epidemiology 1996 University of Chicago press.

[6]. Mukopadhyay C,Bhargava A,Ayyagari A.Two novel clinical presentation of Burkholderia cepacia infection.J Clin Microbiol 2004;42: 3904-5.

[7]. Gautam V,Arora A,Madhup SK ,Das A,Vandamme P,Sharma K, et al.Burkholderia cepacia complex in septicemic non-cystic fibrosis cases from two tertiary care hospitals in NorthIndia .Indian J Med Res 2010;131:829-32.

[8]. Gautam V,Ray P,Das A, Vandamme P,Malhotra P,varma S et al.Two cases of Burkholderia cenocepacia in septicaemic patients .Jpn $\mathrm{J}$ Infect Dis 2008;61:133-4.

[9]. Gautam V,Ray P,Puri GD, ,Sharma K, Vandamme P,Madhup SK, et al .Investigation of Burkholderia cepacia complex in septicaemic patients in a tertiary care hospitals,India ,Nepal Med Coll J. 2009;11:222-4

[10]. Gautam V,Ray P, Vandamme P,Chatterjee SS ,Das A, Sharma K, et al.Identification of lysine positive non-fermenting gram negative bacilli (Stenotrophomonas maltophilia and Burkholderia cepacia complex).Indian J Med Microbiol 2009;27:128-33.

[11]. Clinical Laboratory Standard Institute (CLSI)Performance standard for antimicrobial susceptibility testing .17 informational Supplement ,CLSI M100-S17.Vol. 27 no. 1 Wayne PA :CLSI :2007.

[12]. Martino R,Gomez L,Pericas R,etn al.Bacteremia caused by non glucose fermenting gram negative bacilli and Aeromonas species in patients with haematological malignancies and solid tumours.Eur J Clin Microbiol Infect Dis 2000;19:320-323.

[13]. Zinner S.H.Changing epidemiology of infections in patients with neutropenia and cancer :emphasis on gram positive and resistant bacteria.Clin Infect Dis 1999;29:490-494.

[14]. Donlan RM.Biofilms:Microbial life on surfaces.Emerg Infect Dis 2002;8:881-890.

[15]. Kaslow RA,Mackel DC ,Mallison GF .Nosocomial pseudobacteremia in positive blood culturevdue to contaminated benzalkonium antiseptic.JAMA $1976 ; 236: 2407-2409$.

[16]. Oie S,Kamiya A.Microbial contamination of antiseptics and disinfectants.Am J Infect Control,1996;24: 389-395.

[17]. Centre for Disease control and prevention Nosocomial Burkholderia cepacia infection and colonization associated with intrinsically mouthwash.Arizona 1998 MMWR.

[18]. Dont O,Laul C,Simon AM et al Outbreak of Burkholderia cepacia bacteremia in a paediatric hospital due to contamination of liquid emulsion stoppers.J Clin Microbiol 2004;42:2227-2230.

[19]. Magalhaes M,Doherty C,Govan JR,Vandamme P.Polyclonal outbreak of Burkholderia cepacia complex bacteremia in haemodialysis patients.J Hosp Infect 2003;54:120-123.

[20]. Siddiqui AH,Mulligan ME,Mahenthiralingam F et al An episodic outbreak of genetically related Burkholderia cepacia among noncystic fibrosis patients at university hospital.Infect Control Hosp Epidemiol 2001;22:419-422.

[21]. Aaron SD, Ferris W,Henry DA,Speert DP,Macdonald NE .multiple combination bactericidal antibiotic testing for patients with cystic fibrosis infected with Burkhoideria cepacia.Am J Respir Crit Care Med 2000;161:1206-12.

[22]. Mahenthiralingam E, Baldwin A,Dowson CG.Burkholderia cepacia complex bacteria opportunistic pathogens with important natural biology.J Appl Microbiol 2008;104:1539-51.

[23]. The nonfermentative gram negative bacilli In:Winn W AS,Jande W,Koneman E,Procop G,Schrekernbenger P,Woods G,editors.Konemans colour atlas and Textbook of Diagnostic Microbiology Baltimore, USA:Lippincolt Williams and Wilkins Publisher ;2006.vol- p.303-91.

[24]. Mangram ,Alicia and Jarvis R,William.Nosocomial Burkholderia cepacia outbreaks and pseudo outbreaks .Infection control and hospital Epidemiology Nov 1996 ; Vol 17 (11) :1 sept.2002.

[25]. Levy, Itzhak ,Leibovici, Lenard ,Drucker Moshie,Samra,Zmira,Konisberger ,Hana. A prospective study of gram negative bacteremia in children. The paediatric Infectious Disease Journal .Feb 1996;vol 15:117-122,Nov 2002.

[26]. Maschmeyer G,Mandell : Mandell ,Douglas and Benett's Principle and practice of Infectious Diseases Philadelphia,Pennsylvania :Elsevier Churchill Livingstone ;2009:chapter 220.

[27]. Reik R, Spiker T,Lipuma JJ.Distribution of Burkholderia cepacia complex sepsis among isolates recovered from persons with or without cystic fibrosis .J Clin Microbial 2005;43:2926-8.(PUBMED)(FULL TEXT). 